\title{
Deformations and Liftings of Finite, Commutative Group Scheme
}

\section{Citation}

Oort, Frans, and David B. Mumford. 1968. Deformations and liftings of finite, commutative group scheme. Inventiones Mathematicae 5(4): 317-334.

\section{Published Version}

doi:10.1007/BF01389779

\section{Permanent link}

http://nrs.harvard.edu/urn-3:HUL.InstRepos:3597249

\section{Terms of Use}

This article was downloaded from Harvard University's DASH repository, and is made available under the terms and conditions applicable to Other Posted Material, as set forth at http:// nrs.harvard.edu/urn-3:HUL.InstRepos:dash.current.terms-of-use\#LAA

\section{Share Your Story}

The Harvard community has made this article openly available.

Please share how this access benefits you. Submit a story.

Accessibility 


\section{Deformations and Liftings of Finite, Commutative Group Schemes *}

Frans Oort (Amsterdam) and David Mumford (Cambridge, Mass.)

\section{Introduction}

Consider the following problems:

(A) Given a field $k$, a finite $k$-group scheme $N_{0}$, and a ring $R$ with a surjective ringhomomorphism $R \rightarrow k$. Does there exist a finite, flat $R$-group scheme $N$ such that $N_{0} \cong N \otimes_{R} k$ ? (If so, we say that $N_{0}$ is obtained from $N$ by reduction $\bmod \mathrm{m}$, where $\mathrm{m}=\operatorname{Ker}(R \rightarrow k)$, or, we say that $N$ is a lifting of $N_{0}$ to $R$.)

(B) Given a field $k$ (of characteristic $p>0$ ), and a finite $k$-group scheme $N_{0}$. Does there exist a ring $R$ (integral domain of characteristic zero) with a reduction $R \rightarrow k$, and a finite, flat $R$-group scheme $N$ such that $N_{0} \cong N \otimes_{R} k$ ?

The answers to (A) and to the weaker question (B) are negative in general. However if in (B) moreover is given that $N_{0}$ is a commutative finite group scheme, the answer is affirmative; it is the aim of this paper to give a proof of this fact via deformation theory of finite group schemes in characteristic $p>0$. As a byproduct we obtain a proof for the fact that any finite, local group scheme can be embedded into a formal Lie group with coefficients in the same field, on the same number of parameters.

Example (-A). Let $k$ be a field of characteristic $p>0$ (e.g. the prime field $k=\mathbf{F}_{p}$ ), and let $R$ be a ring with a reduction $R \rightarrow k=R / \mathrm{m}$, such that $p \cdot 1 \notin \mathrm{m}^{2}$ (an "unramified" situation) (e.g. $R=W_{\infty}(k)$, so $W_{\infty}\left(\mathbf{F}_{p}\right)=\mathbf{Z}_{p}$, the ring of $p$-adic integers, or $\left.R=W_{\infty}(k) / p^{2}\right)$. Let $N_{0}=\alpha_{p, k}$, i.e. $N_{0}=\operatorname{Spec}(k[\tau])$, $\tau^{p}=0$, and the group law is defined by $s_{0}: E_{0} \rightarrow E_{0} \otimes_{k} E_{0}, E_{0}=k[\tau]$, with $s_{0}(\tau)=\tau \otimes 1+1 \otimes \tau$; we claim that in this case the answer to problem (A) is negative. Suppose $R$ to be local (localize if necessary), and suppose $N$ as indicated could be found; then $N=\operatorname{Spec}(E), E=R[\sigma]$, where $\sigma^{p}=a_{1} \sigma+\cdots+a_{p-1} \sigma^{p-1}$ with $a_{i} \in \mathfrak{m}$; the group law would be given by some ringhomomorphism $s: E \rightarrow E \bigotimes_{R} E$, so

$$
s(\sigma)=\sigma \otimes 1+1 \otimes \sigma+\sum b_{i j} \sigma^{i} \otimes \sigma^{j}, \quad b_{i j} \in \mathfrak{m} ;
$$

* This work was partially supported by NSF grant GP 3512 .

22 Inventiones math., Vol. 5 
as $(s \sigma)^{p}=s\left(\sigma^{p}\right)$, we obtain:

$$
p \cdot\left(\sigma \otimes \sigma^{p-1}+\cdots+\sigma^{p-1} \otimes \sigma\right) \equiv 0 \quad\left(\bmod m^{2} \cdot E \otimes E\right),
$$

which is a contradiction.

Remark. In the previous situation, by a result of Tate (cf. [13]), we know that $\alpha_{p}$ can be lifted to $R$ (e.g. $R$ is a complete local ring) if and only if $p \in R$ admits a factorization $p=a b$, with $a \in \mathfrak{m}$, and $b \in \mathfrak{m}$.

Example (-B). Let $R$ be an integral domain of characteristic zero, and let $N=\operatorname{Spec}(E)$ be a finite $R$-group scheme such that $E$ is a free $R$-module of rank $p^{2}$ (where $p$ is a prime number). Then $N$ is commutative. This can be seen as follows: let $L$ be an algebraic closure of the field of fractions of $R$; we know that $N \otimes_{R} L$ is reduced (cf. [1], footnote on p.109; cf. [9], lecture 25, theorem 1; cf. [11]), so by group theory it follows that $N \otimes L$, and hence that $N$ is commutative. This shows that any non-commutative group scheme of rank $p^{2}$ cannot be lifted to characteristic zero. It is easy to give an example: take the kernel of the Frobenius homomorphism of a suitable non-commutative linear group. For example, let $N_{0}$ be given by: $k$ is a field of characteristic $p$, and for any $k$-algebra $B$,

$$
\begin{aligned}
& N_{0}(B)=\left\{\text { the multiplicative group of matrices }\left(\begin{array}{ll}
\alpha & \beta \\
0 & 1
\end{array}\right),\right. \\
& \left.\qquad \alpha \in B, \beta \in B, \alpha^{p}=1, \beta^{p}=0\right\} ;
\end{aligned}
$$

so $N_{0}=\operatorname{Spec}\left(E_{0}\right), E_{0}=k[\tau, \rho]$ with $\tau^{p}=1, \rho^{p}=0$, with $s_{0}(\tau)=\tau \otimes \tau$ and $s_{0}(\rho)=\rho \otimes 1+\tau \otimes \rho$.

\section{Liftings of Deformations}

The first example makes it clear that in order to lift a finite (local, unipotent) group scheme to characteristic zero, in general one has to allow ramification at $p$; but it is difficult to obtain directly from $N_{0}$ the information "how much ramification" is needed. Therefore we solve the problem $B$ in the commutative case via deformation theory in characteristic $p>0$. The following lemma is a special case of a general principle: that specializations of liftable "objects" are liftable.

Lemma (2.1). Assume we are given rings: $A \subset K \stackrel{\pi}{\longleftarrow}$, where $R$ is a characteristic zero local domain, $\pi: R \rightarrow R / m=K$ its residue class map, and $A$ a subring of $K$, and that we are given finite free group schemes over these rings

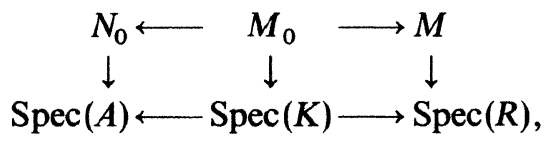


where $M_{0} \cong N_{0} \otimes_{A} K \cong M \otimes_{R} K$. Write $R^{\prime}=\{x \in R \mid \pi(x) \in A\}$; there is a finite free group scheme $N \rightarrow \operatorname{Spec}\left(R^{\prime}\right)$ such that $N_{0} \cong N \otimes_{R^{\prime}} A$ and $M \cong N \otimes_{R^{\prime}} R$.

Proof. Let $N_{0}=\operatorname{Spec}\left(E_{0}\right), M_{0}=\operatorname{Spec}\left(F_{0}\right), M=\operatorname{Spec}(F)$. Then $F_{0} \cong$ $E_{0} \otimes_{A} K \cong F \otimes_{R} K$. Identify $E_{0}$ with the corresponding subset of $F_{0}$, and identify $F_{0}$ with the corresponding quotient of $F$, so $E_{0} \subset F_{0} \stackrel{\pi^{\prime}}{*} F$. Each of these three is a free module of rank $d$, say, over either $A, K$ or $R$, and has the structure of a bialgebra. Let $E=\left\{x \in F \mid \pi^{\prime}(x) \in E_{0}\right\}$, and choose a basis $\left\{b_{1}, \ldots, b_{d}\right\}$ of $E_{0}$ over $k$; let $a_{i} \in F$ satisfy $\pi^{\prime}\left(a_{i}\right)=b_{i}$; one checks easily that $E$ is a free $R^{\prime}$-module with basis $\left\{a_{1}, \ldots, a_{d}\right\}$. Moreover, one can also check

i) that the identity 1 of $F$ is in $E$,

ii) $E$ is closed under multiplication in the ring $F$,

iii) the comultiplication $F \rightarrow F \bigotimes_{R} F$ carries $E$ in $E \otimes_{R^{\prime}} E$,

iv) the augmentation $F \rightarrow R$ carries $E$ in $R^{\prime}$,

v) the inverse $F \rightarrow F$ carries $E$ to $E$.

Therefore $N=\operatorname{Spec}(E)$ is a finite free group scheme over $R^{\prime}$ with all the required properties.

Actually, what we need:

Corollary (2.2). Let $A=k$ be a field, and let $N_{0}$ be a finite $k$-group scheme; this group scheme can be lifted to characteristic zero if and only if for some field extension $k \subset K$ (or for every field extension $k \subset K$ ), $N_{0} \otimes_{k} K$ can be lifted to characteristic zero.

The "if" part follows from (2.1). The "only if" part for example is an easy consequence of the place extension theorem (cf. EGA $0_{\text {III }}, 10.3 .1$ ).

Corollary (2.3). Let $k \leftarrow A \hookrightarrow K$ be ringhomomorphisms, and let $N_{0}=\operatorname{Spec}\left(E_{0}\right)$ be a finite free A-group scheme such that $N_{0} \otimes_{A} K$ can be lifted to characteristic zero. Then $N_{0} \otimes_{A} k$ can be lifted to characteristic zero.

$$
\text { If } N_{0} \cong N \otimes_{R^{\prime}} A \text {, then } N \otimes_{A} k \cong N \bigotimes_{R^{\prime}} A \bigotimes_{A} k \cong N \otimes_{R^{\prime}} k .
$$

\section{Moduli of Rigidified Local Group Schemes}

It is clear that in general the moduli functor for finite group schemes is not representable.

Example. Let char $(k)=p>0$, take $B=k[T]$, and define a $B$-bialgebra by $E=B[\tau]$ with $\tau^{p}=T \tau$ and $s(\tau)=\tau \otimes 1+1 \otimes \tau$; for any field $K \supset k$ and for any $t \in \operatorname{Spec}(B)(K)$ with $t \neq 0$ (i.e. for any $k$-algebra homomorphism $\varphi: B \rightarrow K$ such that $\varphi(T) \neq 0) E_{t}$ is the bialgebra of a reduced $22^{*}$ 
group scheme, isomorphic to $\mathbf{Z} / p$ in case $K$ is algebraically closed, while $E_{0}$ is the bialgebra of the group scheme $\alpha_{p}$.

However by an obvious rigidification of the underlying scheme of the group schemes we can obtain a moduli space. In order to see that any finite group scheme admits a nice deformation we would like to know that this moduli space is irreducible. It is easy to see it is connected, and by imposing extra conditions we can actually obtain a variety.

First we recall the following fact, due to Dieudonné and Cartier. Let $N$ be a finite local $k$-group scheme, where $k$ is a perfect field; $N=$ $\operatorname{Spec}(E)$. Then there exist integers $v_{1}, \ldots, v_{m}$ and an isomorphism

$$
E \cong k\left[X_{1}, \ldots, X_{m}\right] /\left(X_{1}^{p \exp \left(v_{1}\right)}, \ldots, X_{m}^{p \exp \left(v_{m}\right)}\right)
$$

(cf. SGAD, Exp. VII ${ }_{B}, 5.4$; we are writing $p \exp (a)=p^{a}$ for typographical reasons); in this case we say that $E$ admits a truncation type $v=\left(v_{1}, \ldots, v_{m}\right)$.

By the way, the following example shows that in general a finite local group scheme over an imperfect field does not admit a truncation type: let $a \in k, a \notin k^{p}, E=k[X, Y] /\left(X^{p^{2}}, X^{p}-a Y\right)$, and $s(X)=X \otimes 1+$ $1 \otimes X, s(Y)=Y \otimes 1+1 \otimes Y$.

Notation. Let $\alpha=\left(\alpha_{1}, \ldots, \alpha_{m}\right)$ be a set of non-negative integers; we write $X^{\alpha}$ for

$$
X^{\alpha}=X_{1}^{\alpha_{1}} \times \cdots \times X_{m}^{\alpha_{m}}
$$

(with $X_{i}^{0}=1$ ), and we denote by $|\alpha|=\alpha_{1}+\cdots+\alpha_{m}$.

Definition. Let $p$ be a prime number, $v=\left(v_{1}, \ldots, v_{m}\right)$ a set of positive integers, and $\mu=X^{\alpha}$ a monomial in $m$ variables, where $\alpha=\left(\alpha_{1}, \ldots, \alpha_{m}\right)$. We say that $\mu$ satisfies the condition $(P v)_{i}$ for $1 \leqq i \leqq m$, if there exists an index $j$ such that

$$
\alpha_{j} \cdot p^{v_{i}} \geqq p^{v_{j}}
$$

or, equivalently $\left(X^{\alpha}\right)^{p \exp \left(v_{i}\right)}$ is in the ideal generated by $X_{1}^{p \exp \left(v_{1}\right)}, \ldots$, $X_{m}^{p \exp \left(v_{m}\right)}$. We say that a polynomial in $X_{1}, \ldots, X_{m}$ satisfies $(P v)_{i}$ if is can be written as a sum of monomials which all satisfy condition $(P v)_{i}$. We say that a polynomial in the variables $X_{j} \otimes X_{k}, 1 \leqq j \leqq m, 1 \leqq k \leqq m$, satisfies condition $(P v)_{i}$ if it can be written as a sum

$$
\sum_{t} \mu_{1 t} \otimes \mu_{2 t}
$$

where $\mu_{1 t}$ and $\mu_{2 t}$ are monomials such that for each index $t$ either $\mu_{1 t}$ or $\mu_{2 t}$ satisfies $(P v)_{i}$. Analogous definition for a polynomial in the variables $X_{j} \otimes X_{k} \otimes X_{l}$.

Remark. Let $B$ be an integral domain of characteristic $p$, and let $N=\operatorname{Spec}(E)$ be a finite $B$-group scheme, $E=B\left[\tau_{1}, \ldots, \tau_{m}\right]$ with $\tau_{i}^{\text {pexp }\left(v_{i}\right)}=0$, 
$1 \leqq i \leqq m$; the comultiplication is denoted by $s: E \rightarrow E \otimes E$. As $s$ is a ringhomomorphism it follows that $\left(s \tau_{i}\right)^{p \exp \left(v_{i}\right)}=0$, so $s\left(\tau_{i}\right)$ is a polynomial in $\tau_{j} \otimes \tau_{k}$ which satisfies condition $(P v)_{i}$. The same for the polynomials $\gamma\left(\tau_{i}\right)$, where $\gamma: E \rightarrow E$ defines the inverse.

We fix $k$, a field of characteristic $p>0$, and $v=\left(v_{1}, \ldots, v_{m}\right)$, a set of positive integers; $\mathbf{C}=\mathrm{C}_{\boldsymbol{k}}$ denotes the category of $k$-algebras. Define a functor $\Sigma_{v}=\Sigma: \mathbf{C} \rightarrow$ Ens by:

$\Sigma(B)=\left\{\right.$ all cocommutative $B$-bialgebra structures on $B\left[\tau_{1}, \ldots, \tau_{m}\right]=E$, such that $s\left(\tau_{i}\right)$ are polynomials satisfying condition $(P v)_{i}$ for $1 \leqq i \leqq m\}$,

where $\tau_{i}^{p \exp \left(v_{i}\right)}=0$ for $1 \leqq i \leqq m$, and where the augmentation ideal of $E$ is generated by $\tau_{1}, \ldots, \tau_{m}$. Note that a $B$-bialgebra $F$ can correspond to various elements of $\Sigma(B)$, as there may exist several isomorphisms $F \cong B\left[\tau_{1}, \ldots, \tau_{m}\right]$.

Theorem (3.1). We fix $k$, and $v=\left(v_{1}, \ldots, v_{m}\right)$ as before; the functor $\Sigma: \mathbf{C} \rightarrow$ Ens is represented by a k-algebra $U$, and there exists an integer $n$ such that $U \cong k\left[T_{1}, \ldots, T_{n}\right]$.

It is easy to see that $\Sigma$ is representable; however the first step of the proof will be more complicated as we want to obtain information for late use.

Proof, first step: $\Sigma$ is representable. Consider all combinations $\left(i, \alpha=\left(\alpha_{1}, \ldots, \alpha_{m}\right), \beta=\left(\beta_{1}, \ldots, \beta_{m}\right)\right)$ such that $1 \leqq i \leqq m, 0 \leqq \alpha_{j}<p \exp \left(v_{j}\right)$, $0 \leqq \beta_{j}<p \exp \left(v_{j}\right)$, and such that the monomial $\tau^{\alpha} \otimes \tau^{\beta}$ satisfies condition $(P v)_{i}$ (i. e. either $\left(\tau^{\alpha}\right)^{p \exp \left(v_{i}\right)}=0$, or $\left.\left(\tau^{\beta}\right)^{p \exp \left(v_{i}\right)}=0\right)$, and such that $|\alpha|>0$ and $|\beta|>0$; let $A=k\left[\ldots, Y_{i, \alpha, \beta}, \ldots\right]$, and let $F=A\left[\tau_{1}, \ldots, \tau_{m}\right]$ with $\tau_{i}^{p \exp \left(v_{i}\right)}=0,1 \leqq i \leqq m$. Then we are given an $A$-algebra homomorphism

by

$$
s: F \rightarrow F \otimes_{A} F
$$

$$
s\left(\tau_{i}\right)=\tau_{i} \otimes 1+1 \otimes \tau_{i}+\sum_{\alpha, \beta} Y_{i, \alpha, \beta} \tau^{\alpha} \otimes \tau^{\beta}
$$

( $s$ is a ringhomomorphism because of the conditions $(P v)_{i}$, but this is not the point where these conditions are used essentially). Let $\mu_{1}$, $\mu_{2}, \ldots$ be all non-zero monomials of the form $\tau^{\alpha} \otimes \tau^{\beta} \otimes \tau^{\gamma}$; we write $\Gamma s=(s \otimes 1) \cdot s-(1 \otimes s) \cdot s$, and

$$
(\Gamma s)\left(\tau_{i}\right)=\sum_{j} H_{i j} \mu_{j}, \quad 1 \leqq i \leqq m,
$$

with $H_{i j} \in A$; let $\mathfrak{p} \subset A$ be the ideal generated by these polynomials, and by the symmetry relations:

$$
\mathfrak{p}=\left(\ldots, H_{i j}, \ldots, \ldots, Y_{i, \alpha, \beta}-Y_{i, \beta, \alpha}, \ldots\right) \cdot A .
$$


We define $U=A / \mathfrak{p}$, and $E=U\left[\tau_{1}, \ldots, \tau_{m}\right]$. It is clear that $s$ induces a coassociative comultiplication

defined by

$$
s: E \rightarrow E \otimes_{U} E,
$$

$$
s\left(\tau_{i}\right)=\tau_{i} \otimes 1+1 \otimes \tau_{i}+\sum_{\alpha, \beta} y_{i, \alpha, \beta} \tau^{\alpha} \otimes \tau^{\beta},
$$

where $y_{i, \alpha, \beta}=Y_{i, \alpha, \beta} \bmod \mathfrak{p}$. Clearly the pair $(U, E)$ represents the functor $\Omega_{m, v}=\Omega$ defined by:

$\Omega(B)=\{$ all cocommutative coassociative $B$-algebra homomorphisms $s: E \rightarrow E \otimes_{B} E$, where $E=B\left[\tau_{1}, \ldots, \tau_{m}\right]$, such that $s(x) \equiv x \otimes 1+$ $1 \otimes x(\bmod \mathfrak{a} \otimes \mathfrak{a}), \mathfrak{a}=\left(\tau_{1}, \ldots, \tau_{m}\right) \cdot E$, and such that $s\left(\tau_{i}\right)$ satisfies condition $(P v)_{i}$ for $\left.1 \leqq i \leqq m\right\}$.

The following lemma asserts that $\Sigma(B) \rightarrow \Omega(B)$ :

Lemma (3.2). Let $B$ be a ring in which $p \cdot 1=0$, let $E=B\left[\tau_{1}, \ldots, \tau_{m}\right]$ with $\tau_{i}^{p \exp \left(v_{i}\right)}=0,1 \leqq i \leqq m$, and with augmentation ideal $\mathfrak{a}=\left(\tau_{1}, \ldots, \tau_{m}\right) \cdot E$. Let s: $E \rightarrow E \otimes_{B} E$ be a B-algebra homomorphism such that

$$
s(x) \equiv x \otimes 1+1 \otimes x \quad(\bmod \mathfrak{a} \otimes \mathfrak{a})
$$

for all $x \in \mathfrak{a}$ (i.e. the augmentation is a left-and a right-coidentity), and such that $s\left(\tau_{i}\right)$ satisfies condition $(P v)_{i}$ for $1 \leqq i \leqq m$. Then there exists a unique B-algebra homomorphism $\gamma: E \rightarrow E$ such that $m(\gamma \otimes 1) s(x)=0$ for all $x \in \mathfrak{a}$ (where $m: E \bigotimes_{B} E \rightarrow E$ is the multiplication).

Proof. We define $\gamma_{1}\left(\tau_{i}\right)=-\tau_{i}$; thus we have defined a $B$-algebra homomorphism $\gamma_{1}: E \rightarrow E$ having the property

$$
m\left(\gamma_{1} \otimes 1\right) s(x) \in \mathfrak{a}^{2} \quad \text { for all } x \in \mathfrak{a},
$$

and it is unique modulo $\mathfrak{a}^{2}$ among all having this property. Suppose for some $N \geqq 1$ there is given a $B$-algebra homomorphism $\gamma_{N}: E \rightarrow E$ such that

$$
m\left(\gamma_{N} \otimes 1\right) s(x)=\rho_{N}(x) \in \mathfrak{a}^{N+1} \quad \text { for all } x \in \mathfrak{a},
$$

and such that $\gamma_{N}\left(\tau_{i}\right)$ satisfies condition $(P v)_{i}$ for $1 \leqq i \leqq m$. It is easy to see that $\rho_{N}\left(\tau_{i}\right)$ satisfies condition $(\boldsymbol{P} v)_{i}$; thus

$$
\gamma_{N+1}\left(\tau_{i}\right)=\gamma_{N}\left(\tau_{i}\right)-\rho_{N}\left(\tau_{i}\right), \quad 1 \leqq i \leqq m,
$$

defines a $B$-algebra homomorphism $\gamma_{N+1}: E \rightarrow E$; it is clear that

$$
m\left(\gamma_{N+1} \otimes 1\right) s\left(\tau_{i}\right) \in \mathfrak{a}^{N+2} \quad \text { for } 1 \leqq i \leqq m,
$$

and it is readily verified that if $\gamma^{\prime}$ also has the property $m\left(\gamma^{\prime} \otimes 1\right) s(x) \in \mathfrak{a}^{N+2}$ for all $x \in \mathfrak{a}$, and $\gamma^{\prime}\left(\tau_{i}\right)-\gamma_{N+1}\left(\tau_{i}\right) \in \mathfrak{a}^{N+1}$ for all $i$, then $\gamma^{\prime}(x) \equiv \gamma_{N+1}(x)(\bmod$ 
$\mathfrak{a}^{N+2}$ ) for all $x \in \mathfrak{a}$. Thus the construction of $\gamma$ and its uniqueness follow by induction as $\mathfrak{a}^{|\gamma|}=0$.

Thus the ring $U$ and the bialgebra structure on $E$ represent the functor $\Sigma \cong \Omega$, and the first step of the proof is concluded. Let $W=$ $\operatorname{Spec}(U)$; consider the point $0 \in W(k)$ defined by $y_{i, \alpha, \beta} \mapsto 0$, i.e. $s\left(\tau_{i}\right)=$ $\tau_{i} \otimes 1+1 \otimes \tau_{i}$ and $\gamma\left(\tau_{i}\right)=-\tau_{i}$; that is the point corresponding to the rigidified group scheme $\alpha_{p \exp \left(v_{1}\right)} \times \cdots \times \alpha_{p \exp \left(v_{m}\right)}$.

The crucial part of the proof of the theorem is: $0 \in W(k)$ is a nonsingular point of $W$ (note that this is false if $W$ were the moduli space of all rigidified group schemes, say of a fixed rank, not necessarily local; note that this is also false if $W$ were the moduli space of all rigidified local group schemes, not all the $v_{i}$ equal, and not imposing the extra conditions $\left.(P v)_{i}\right)$. This we can show in two ways. It can be deduced from results of Lazard about formal group laws; this will be done in the next section. We could also have used the group-cohomology as described in SGAD, Exp. III, especially p. III. 42/43, Theorem 3.5 (also cf. [8]), and using a result of G. Efroymson, which says that $H_{\text {symm }}^{3}\left(N, \mathbf{G}_{a}\right)=0$ (trivial action of the commutative finite group scheme $N$ on the additive linear group $\mathbf{G}_{a}$ ) (proved in his Harvard thesis, 1966, later generalized into a structure theorem about the cohomology ring $H^{*}\left(N, \mathbf{G}_{a}\right)$, not yet published).

\section{Finite Group Schemes and Buds}

First we recall some definitions and results to be found in a paper by Lazard, cf. [5]. Let $m$ and $r$ be positive integers, $R$ a ring (commutative, and $1 \in R$ ), and

$$
f: R\left[X_{1}, \ldots, X_{m}\right]=E \rightarrow E \otimes_{R} E
$$

an $R$-algebra homomorphism; we say that $f$ defines an $r$-bud (" $r$ bourgeon") on $m$ parameters, with coefficients in $R$ if (we write $(f \otimes 1) \cdot f-$ $(1 \otimes f) \cdot f=\Gamma f)$ :

$$
(\Gamma f)\left(X_{i}\right) \equiv 0 \quad(\bmod \text { degree } r+1) \text { for } 1 \leqq i \leqq m
$$

(degree means total degree in the variables $\left.X_{1} \otimes 1, \ldots, 1 \otimes X_{m}\right) ; f$ and $g$ define the same $r$-bud if and only if $f\left(X_{i}\right) \equiv g\left(X_{i}\right)$ (mod degree $\left.r+1\right)$ for $1 \leqq i \leqq m$ (cf. [5], p.381, Definition 13.1); a system $f_{1}, f_{2}, \ldots$ such that $f_{r}$ is an $r$-bud on $m$ parameters, and such that $f_{r}$ and $f_{r+1}$ define the same $r$-bud is called a formal Lie group on $m$ parameters. We write $\Lambda_{m, r}(R)=\Lambda(R)=$ all cocommutative $r$-buds ("r-bourgeons abéliens") on $m$ parameters with coefficients in $R\}$;

clearly we have thus obtained a covariant functor $\Lambda_{m, r}$ defined on the category of commutative rings with identity; if $f \in \Lambda_{m, r}(E)$ and $\varphi: E \rightarrow R$ 
is a ring homomorphism we write $(\Lambda \varphi)(f) \in \Lambda_{m, r}(R)$ for the $r$-bud over $R$ obtained from $f$, applying $\varphi$. Lazard has proved:

(i) (cf. [5], pp.394-399, and previous pages). Let

$$
N(m, r)=N=m\left(\left(\begin{array}{c}
r+m \\
\mathrm{~m}
\end{array}\right)-m-1\right) ;
$$

there exists a universal

$$
F_{r} \in \Lambda_{m, r}\left(A_{r}\right), \quad A_{r}=\mathbf{Z}\left[T_{1}, \ldots, T_{N(m, r)}\right],
$$

i.e. $\left(A_{r}, F_{r}\right)$ represents the functor $\Lambda_{m, r}$, or: the map

$$
\operatorname{RHom}\left(A_{r}, \mathrm{R}\right) \rightarrow \Lambda_{m, r}(R)
$$

defined by $\varphi \mapsto(\Lambda \varphi)\left(F_{r}\right)$ is bijective for every $R$.

(ii) The natural restriction map $\Lambda_{m, r+1}(R) \rightarrow \Lambda_{m, r}(R)$ is surjective if $R$ is without integral torsion (cf. [5], p.396, Lemma 15.2), hence, by (i), this map is surjective for every $R$; it corresponds to the inclusion map

$$
A_{r}=\mathbf{Z}\left[T_{1}, \ldots, T_{N(m, r)}\right] \rightarrow A_{r+1}=\mathbf{Z}\left[T_{1}, \ldots, T_{N(m, r+1)}\right],
$$

such that $F_{r} \in \Lambda_{r}\left(A_{r}\right) \subset \Lambda_{r}\left(A_{r+1}\right)$ and $F_{r+1} \in \Lambda_{r+1}\left(A_{r+1}\right)$ define the same $r$-bud.

(iii) Suppose $f_{r}$ and $f_{r+1}$ define the same $r$-bud on $m$ parameters with coefficients in $R ;\left(\Lambda \varphi_{r}\right)\left(F_{r}\right)=f_{r}$ and $\left(\Lambda \varphi_{r+1}\right)\left(F_{r+1}\right)=f_{r+1}$; then the diagram

commutes. Hence

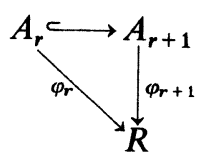

$$
A=\bigcup A_{r}=\mathbf{Z}\left[T_{1}, T_{2}, \ldots\right]
$$

represents the functor of all formal Lie groups on $m$ parameters (cf. [5], p.397, Theorem 15.1); in particular, any $r$-bud on $m$ parameters can be extended to a formal Lie group on $m$ parameters with coefficients in the same ring.

Suppose we fix $k$, a field of characteristic $p>0$, a positive integer $m$, and positive integers $v_{1}, \ldots, v_{m}$. We choose an integer $r$ so that

$$
r \geqq 3 \cdot \sum_{i=1}^{m}\left(p \exp \left(v_{i}\right)-1\right) \text {. }
$$

We consider only rings $R$ containing $k$, in particular $p \cdot 1=0$ in $R$. We restrict the functor $\Lambda$ to the category of $k$-algebras; for such rings we define a functor $\Delta$ by:

$$
\Delta_{m, r, v}=\Delta \subset \Lambda_{m, r}
$$

$\Delta(R)=\left\{f \in \Lambda_{m, r}(R)\right.$ such that $f\left(X_{i}\right)$ satisfies condition $(P v)_{i}$ for $\left.1 \leqq i \leqq m\right\}$. 
For $f \in \Delta(R)$, we define $\rho(f)$ by

$$
\rho(f)\left(\tau_{i}\right)=f\left(X_{i}\right) \bmod \left(X_{1}^{p \exp \left(v_{1}\right)}, \ldots, X_{m}^{p \exp \left(v_{m}\right)}\right) ;
$$

because of the conditions $(P v)_{i}$ we thus obtain an $R$-algebra homomorphism (!)

$$
\rho(f): E \rightarrow E \otimes_{R} E, \quad E=R\left[\tau_{1}, \ldots, \tau_{m}\right],
$$

where $\tau_{i}^{p \exp \left(v_{i}\right)}=0,1 \leqq i \leqq m$, and because of the choice of $r$ it follows that

$$
(\Gamma s)\left(\tau_{i}\right)=0, \quad 1 \leqq i \leqq m,
$$

so $\rho(f) \in \Omega(R)$ (in the notation introduced in Section 3). So we have the following morphisms of functors (defined on $k$-algebras):

$$
\Sigma \cong \Omega_{m, v}=\Omega \leftarrow \Delta_{m, r, v} \subset \Lambda_{m, r} .
$$

Proposition (4.1). We fix $k, m, v_{1}, \ldots, v_{m}$, and $r \geqq 3 \cdot \sum\left(p \exp \left(v_{i}\right)-1\right)$ as before. The functors

$$
\Lambda, \Delta, \Omega: \mathbf{C} \rightarrow \text { Ens }
$$

are representable, say by $L, D$, and $W$. The schemes $D$ and $W$ (and also $L$ ) are isomorphic to affine spaces over $k$. In suitable coordinates the morphism $\rho: D \rightarrow W$ is given by a projection

$$
D \cong \operatorname{Spec}\left(k\left[T_{1}, \ldots, T_{n}, T_{1}^{\prime}, \ldots, T_{m}^{\prime}\right]\right) \rightarrow \operatorname{Spec}\left(k\left[T_{1}, \ldots, T_{n}\right]\right) \cong W ;
$$

in particular, for every $R \supset k$ the map $\rho: D(R) \rightarrow W(R)$ is surjective.

In order to deduce these facts from Lazard's results, we need the following tools:

Lemma (4.2). Let

$$
f\left(X_{i}\right)=\sum_{\alpha, \beta} a_{i, \alpha, \beta} X^{\alpha} \otimes X^{\beta}
$$

be polynomials with coefficients in a ring $R$ with $p \cdot 1=0$, such that $f\left(X_{i}\right)$ satisfies condition $(P v)_{i}, 1 \leqq i \leqq m ;$ then $(f \otimes 1) f\left(X_{i}\right)$, and also $(1 \otimes f)$ $f\left(X_{i}\right)$, can be written as a sum of monomials satisfying condition $(P v)_{i}$.

Proof.

$$
(f \otimes 1) f\left(X_{i}\right)=\sum_{\alpha, \beta} a_{i, \alpha, \beta}\left\{\prod_{j}\left[\sum a_{j, \gamma, \delta} X^{\gamma} \otimes X^{\delta}\right]^{\alpha_{j}}\right\} \otimes X^{\beta}=\sum_{\alpha, \beta} a_{i, \alpha, \beta} Q_{i, \alpha, \beta} .
$$

It suffices to consider each $Q_{i, \alpha, \beta}$ separately; either $X^{\beta}$ satisfies condition $(P v)_{i}$, and we are done, or there exists an index $e$ such that $\alpha_{e} \cdot p \exp \left(v_{i}\right) \geqq p \exp \left(v_{e}\right)$, so $p \exp \left(n+v_{i}\right) \geqq p \exp \left(v_{e}\right)$ with $\alpha_{e} \geqq p^{n}$, and $n \geqq 0$; in that case

$$
\begin{aligned}
Q_{i, \alpha, \beta} & =\left\{\left[\sum a_{e, \gamma, \delta} X^{\gamma} \otimes X^{\delta}\right]^{p^{n}} \times(\cdots)\right\} \otimes X^{\beta} \\
& =\left\{\left\{\sum\left[a_{e, \gamma, \delta} X^{\gamma} \otimes X^{\delta}\right]^{p^{n}}\right\} \times(\cdots)\right\} \otimes X^{\beta}
\end{aligned}
$$


for each $(e, \gamma, \delta)$ there exists an index $d$ such that $\gamma_{d} \cdot p \exp \left(v_{e}\right) \geqq p \exp \left(v_{d}\right)$, or $\delta_{d} \cdot p \exp \left(v_{e}\right) \geqq p \exp \left(v_{d}\right)$, hence

$$
p^{n} \cdot \gamma_{d} \cdot p \exp \left(v_{i}\right) \geqq \gamma_{d} \cdot p \exp \left(v_{e}\right) \geqq p \exp \left(v_{d}\right),
$$

or the same with $\delta_{d}$, and $\left(Q_{i, \alpha, \beta}\right)^{p \exp \left(v_{i}\right)}$ is divisable by $\left(X_{d} \otimes 1 \otimes 1\right)^{p \exp \left(v_{d}\right)}$, respectively divisable by $\left(1 \otimes X_{\alpha} \otimes 1\right)^{p \exp \left(\gamma_{\alpha}\right)}$, and the lemma is proved.

Lemma (4.3). Let $R$ be a ring, $M$ an ideal in $R$, and $b \in R$ so that $M \cdot b=0$. Let $E=R\left[X_{1}, \ldots, X_{m}\right]$, and $g: E \rightarrow E \otimes E$ so that

$$
g\left(X_{i}\right) \equiv X_{i} \otimes 1+1 \otimes X_{i} \quad(\bmod M \cdot E \otimes E) .
$$

Let $P=b X^{\alpha} \otimes X^{\beta}$ be a monomial such that $X^{\alpha}$ and $X^{\beta}$ do not satisfy condition $(P v)_{i}$ (for some fixed index $\left.i\right)$; then $(g \otimes 1)(P)$, and also $(1 \otimes g)(P)$, can be written as a sum of monomials none of which satisfy condition $(P v)_{i}$.

Proof.

$(g \otimes 1)(P)=b \cdot g\left(X^{\alpha}\right) \otimes X^{\beta}=b \cdot\left\{\prod_{j}\left(X_{j} \otimes 1+1 \otimes X_{j}\right)^{\alpha_{j}}\right\} \otimes X^{\beta}$

as $M \cdot b=0$, and the lemma is proved.

Let $k$ be a field, $W$ a $k$-algebraic scheme, and $w \in W(k)$. The following statements are known to be equivalent:

(i) $w$ is a non-singular point on $W$;

(ii) the local ring $\mathcal{O}$ of $w$ on $W$ is a regular local ring, i.e. its completion $\hat{\mathcal{O}}$ is a formal power series ring $\hat{\mathcal{O}} \cong k \llbracket e_{1}, \ldots, e_{n} \rrbracket$;

(iii) (Grothendieck's criterion, cf. SGA, III.3.1 and II.5.10) for every local artinian $k$-algebra $R$, maximal ideal $M$, and any ideal $I \subset R$ so that $M \cdot I=0$, the map $W(R)_{w} \rightarrow W(R / I)_{w}$ is surjective (we write $W(R)_{w}$ for the set of morphisms $W \rightarrow \operatorname{Spec}(R)$ with $(W \rightarrow \operatorname{Spec}(R) \rightarrow \operatorname{Spec}(k))=w)$.

Lemma (4.4). Let $\rho: D \rightarrow W$ be a morphism of k-algebraic schemes, and $d \in D(k)$ a non-singular point on $D$; suppose the tangential map

$$
\rho_{*}: t_{D, d} \rightarrow t_{W, \rho(d)}
$$

to be surjective. Then $\rho(d)=w \in W(k)$ is a non-singular point on $W$.

Proof. Let $e_{1}, \ldots, e_{n} \in \mathcal{O}_{W, w}$ be choosen in such a way that their residues modulo $\mathrm{m}^{2}$ form a $k$-base for $\mathrm{m} / \mathrm{m}^{2}$, where $\mathrm{m}$ is the maximal ideal of $\mathcal{O}_{W, w}$. We obtain:

$$
k \llbracket e_{1}, \ldots, e_{n} \rrbracket \stackrel{\pi}{\longrightarrow} \hat{\mathcal{O}}_{W, w} \stackrel{\varphi}{\longrightarrow} \hat{\mathcal{O}}_{D, d} ;
$$

as the tangential map $\rho_{*}$ is surjective, the images of the $e_{i}$ 's are linearly independent modulo the square of the maximal ideal of $\mathcal{O}_{D, d}$; as $d$ is 
a non-singular point this implies that the composition $\varphi \cdot \pi$ is injective; thus $\pi$ is injective (and it is also surjective), so $\hat{\mathcal{O}}_{W, w}$ is a formal power series ring, hence $w \in W(k)$ is a non-singular point, and the lemma is proved.

Elimination Lemma (4.5). Let $A=k\left[T_{1}, \ldots, T_{N}\right]$, and $H_{1}, \ldots, H_{d} \in A$. Suppose given positive integers $w\left(T_{1}\right), \ldots, w\left(T_{N}\right)$ such that $H_{1}, \ldots, H_{d}$ are homogeneous polynomials in the weighed variables $T_{1}, \ldots, T_{n}$ (i.e. we write $w\left(\prod T_{n_{i}}\right)=\sum w\left(T_{n_{i}}\right)$; if $\mu_{1}$ and $\mu_{2}$ are monomials occuring with non-zero coefficients in some $H_{j}$, then $\left.w\left(\mu_{1}\right)=w\left(\mu_{2}\right)\right)$. Suppose $H_{1}(0)=$ $0=H_{2}(0)=\cdots=H_{d}(0)$, such that 0 is a non-singular point of $V=\operatorname{Spec}(A /$ $\left.\left(H_{1}, \ldots, H_{d}\right) A\right)$. Then we can renumber the variables, and we can choose $0 \leqq n \leqq N$ so that

$$
A /\left(H_{1}, \ldots, H_{d}\right) A \cong k\left[T_{1}, \ldots, T_{n}\right] .
$$

Proof. Suppose $\left(H_{1}, \ldots, H_{d}\right) A \neq 0$ (otherwise the conclusion is obvious); in that case at least one of these polynomials has a linear term: if not, we would have

so

$$
\left(H_{1}, \ldots, H_{d}\right) A \subset\left(T_{1}^{2}, \ldots, T_{i} T_{j}, \ldots, T_{N}^{2}\right) A=\mathfrak{b},
$$

$$
\operatorname{Spec}(A / \mathfrak{b}) \subset V \varsubsetneqq \mathbf{A}_{k}^{N}=\operatorname{Spec}\left(k\left[T_{1}, \ldots, T_{N}\right]\right),
$$

a contradiction with the fact that $0 \in V(k)$ is non-singular. So let

$$
H_{d}=c T_{N}+G, \quad c \in k, c \neq 0
$$

so that $T_{N}$ does not appear in the linear term of $G$ (renumber the variables and the polynomials if necessary); as $w\left(T_{i}\right)$ are positive integers for all $i$, it follows that $G \in k\left[T_{1}, \ldots, T_{N-1}\right]$. We write

and clearly

$$
G_{i}=H_{i}\left(T_{1}, \ldots, T_{N-1},-\frac{1}{c} G\left(T_{1}, \ldots, T_{N-1}\right)\right), \quad 1 \leqq i<d,
$$

$$
A /\left(H_{1}, \ldots, H_{d}\right) \cong k\left[T_{1}, \ldots, T_{N-1}\right] /\left(G_{1}, \ldots, G_{d-1}\right)
$$

(the variable $T_{N}$ is eliminated); moreover it is clear that the polynomials $G_{1}, \ldots, G_{d-1}$ are homogeneous in the weighed variables $T_{1}, \ldots, T_{N-1}$; thus the lemma is proved by induction on $d$.

Proof of Proposition (4.1). We proved that $\Omega$ is represented by $W$ in Section 3, by the results of Lazard we know $\Lambda$ is representable, and it is easy to see that $\Delta$ is representable (cf. below). The point $0 \in D(k)$ is defined by $f \in \Delta(k), f\left(X_{i}\right)=X_{i} \otimes 1+1 \otimes X_{i}$; first we show that this is a non-singular point on $D$. Let $R$ be a local artinian $k$-algebra, with maximal ideal $M$, and let $I \subset R$ be an ideal such that $M \cdot I=0$; we write 
$R^{\prime}=R / I$. By Grothendieck's criterion it suffices to show that

$$
D(R)_{0} \rightarrow D\left(R^{\prime}\right)_{0}
$$

is a surjective map. Thus given $f^{\prime} \in \Delta\left(R^{\prime}\right)_{0}=D\left(R^{\prime}\right)_{0}$, we would like to construct $f \in \Delta(R)_{0}$ so that $f^{\prime} \equiv f(\bmod (I \cdot E \otimes E))\left(\right.$ where $E=k\left[X_{1}, \ldots\right.$, $\left.X_{m}\right]$ ); by the result of Lazard we know that $\Lambda$ is represented by a nonsingular scheme (in fact affine space of dimension $N(m, r)$ ), so for $f^{\prime} \in \Delta\left(R^{\prime}\right)_{0} \subset \Lambda\left(R^{\prime}\right)_{0}$ there exists a $g \in \Lambda(R)_{0}$ so that

We know that

$$
f^{\prime} \equiv g \quad(\bmod I \cdot E \otimes E) .
$$

$$
g\left(X_{i}\right) \equiv X_{i} \otimes 1+1 \otimes X_{i} \quad(\bmod M \cdot E \otimes E),
$$

as we work in the point $0 \in D(k) \subset L(k)$; we write

$$
g\left(X_{i}\right)=f\left(X_{i}\right)+c\left(X_{i}\right)
$$

where $c\left(X_{i}\right)$ consists of monomials none of which satisfy condition $(P v)_{i}$, and $f\left(X_{i}\right)$ consists of monomials which satisfy condition $(P v)_{i}$. We claim that

$$
(\Gamma f) \equiv 0 \quad(\bmod \text { degree } r+1),
$$

i.e. $f \in \Delta(R)_{0}$; in fact let

$$
\begin{aligned}
& f\left(X_{i}\right)=X_{i} \otimes 1+1 \otimes X_{i}+\sum_{\alpha, \beta} a_{i, \alpha, \beta} X^{\alpha} \otimes X^{\beta}, \\
& c\left(X_{i}\right)=\sum_{\alpha, \beta} b_{i, \alpha, \beta} X^{\alpha} \otimes X^{\beta} ;
\end{aligned}
$$

then $a_{i, \alpha, \beta} \in M$ and $b_{i, \alpha, \beta} \in I$. Using $M \cdot I=0$, we obtain:

$$
\begin{aligned}
(g \otimes 1) g\left(X_{i}\right)= & {\left[(f \otimes 1) f\left(X_{i}\right)\right] } \\
& +\left[\sum_{\alpha, \beta} b_{i, \alpha, \beta} X^{\alpha} \otimes X^{\beta} \otimes 1+(g \otimes 1)\left(\sum_{\alpha, \beta} b_{i, \alpha, \beta} X^{\alpha} \otimes X^{\beta}\right)\right] .
\end{aligned}
$$

By (4.2) the first term in square brackets can be written as a sum of monomials all satisfying condition $(P v)_{i}$; by $(4.3)$ the second term can be written as a sum of monomials none of which satisfy condition $(P v)_{i}$. Thus the equation $(\Gamma g)\left(X_{i}\right) \equiv 0(\bmod$ degree $r+1)$ proves, by sorting out all $(P v)_{i}$-monomials, that

$$
(\Gamma f)\left(X_{i}\right) \equiv 0 \quad(\bmod \text { degree } r+1),
$$

thus $f \in \Delta(R)_{0}$, and we have proved that $0 \in D(k)$ is a nonsingular point on $D$.

Next we show that $0 \in W$ is a non-singular point on $W$. Let $R=k[\varepsilon]$, with $\varepsilon^{2}=0$. We know that $t_{D, 0}=\Delta(k[\varepsilon])_{0}$, hence by (4.4) it suffices 
to show that

$$
\rho_{*}: \Delta(k[\varepsilon])_{0} \rightarrow \Omega(k[\varepsilon])_{0}
$$

is a surjective map. Hence we are given

with

$$
s: E \rightarrow E \otimes E, \quad E=R\left[\tau_{1}, \ldots, \tau_{m}\right],
$$

$$
s\left(\tau_{i}\right)=\tau_{i} \otimes 1+1 \otimes \tau_{i}+\varepsilon \cdot \sum c_{i, \alpha, \beta} \tau^{\alpha} \otimes \tau^{\beta}, \quad c_{i, \alpha, \beta} \in k,
$$

satisfying $(P v)_{i}$ and $(\Gamma s)=0$, and we have to construct an $r$-bud $f$ satisfying again the conditions $(P v)_{i}$ extending $s$. We choose

$$
f\left(X_{i}\right)=X_{i} \otimes 1+1 \otimes X_{i}+\varepsilon \cdot \sum c_{i, \alpha, \beta} X^{\alpha} \otimes X^{\beta} ;
$$

as $\varepsilon^{2}=0$, we obtain

$$
\begin{aligned}
(f \otimes 1) f\left(X_{i}\right)= & X_{i} \otimes 1 \otimes 1+1 \otimes X_{i} \otimes 1+1 \otimes 1 \otimes X_{i} \\
& +\varepsilon \cdot \sum c_{i, \alpha, \beta} X^{\alpha} \otimes X^{\beta} \otimes 1 \\
& +\varepsilon \cdot \sum c_{i, \alpha, \beta}\left\{\prod_{j}\left(X_{j} \otimes 1+1 \otimes X_{j}\right)^{\alpha_{j}}\right\} \otimes X^{\beta}
\end{aligned}
$$

in each of these terms the exponent of $X_{j}$ is smaller than $p \exp \left(v_{j}\right)$, thus $\Gamma s=0$ proves that $(\Gamma f)\left(X_{i}\right)=0$. Thus $f \in \Delta(R)_{0}$, and certainly $\rho(f)=s$, and we have shown the tangential map $\rho_{*}$ to be surjective; as $0 \in D$ is a non-singular point we conclude by (4.4) that $0 \in W$ is non-singular.

Now we prove that $D$ and $W$ are isomorphic to affine spaces over $k$. Let $\Delta^{\prime}$ be the set of pairs $(\alpha, \beta)$ with $\alpha=\left(\alpha_{1}, \ldots, \alpha_{m}\right), \beta=\left(\beta_{1}, \ldots, \beta_{m}\right)$ so that $1 \leqq|\alpha|$ and $1 \leqq|\beta|$ and $|\alpha|+|\beta| \leqq r$; let $\Delta^{\prime \prime}$ be the set of triples $(\alpha, \beta, \gamma)$ with $1 \leqq|\alpha|, 1 \leqq|\beta|, 1 \leqq|\gamma|$, and $|\alpha|+|\beta|+|\gamma| \leqq r$. Let $\Omega^{\prime}$ be the set of pairs $(\alpha, \beta)$ with $1 \leqq|\alpha|$ and $0 \leqq \alpha_{j}<p \exp \left(v_{j}\right)$ for $1 \leqq j \leqq m$, and $1 \leqq|\beta|$ and $0 \leqq \beta_{k}<p \exp \left(v_{k}\right)$ for $1 \leqq k \leqq m$; let $\Omega^{\prime \prime}$ be the set of triples $(\alpha, \beta, \gamma)$ with $1 \leqq|\alpha|$ and $0 \leqq \alpha_{j}<p \exp \left(v_{j}\right)$, etc. Consider

$$
F\left(X_{i}\right)=X_{i} \otimes 1+1 \otimes X_{i}+\sum_{\alpha, \beta} T_{i, \alpha, \beta} X^{\alpha} \otimes X^{\beta},
$$

summation taken over all $(\alpha, \beta) \in \Delta^{\prime}$, respectively summation taken over all $(\alpha, \beta) \in \Omega^{\prime}$; we write $k\left[\Delta^{\prime}\right]$, resp. $k\left[\Omega^{\prime}\right]$, for the polynomial ring $k\left[\ldots, T_{i, \alpha, \beta}, \ldots\right], 1 \leqq i \leqq m$ and $(\alpha, \beta) \in \Delta^{\prime}$, resp. $1 \leqq i \leqq m$ and $(\alpha, \beta) \in \Omega^{\prime}$. We define polynomials $H_{i, \alpha, \beta, \gamma} \in k\left[\Delta^{\prime}\right]$, resp. $H_{i, \alpha, \beta, \gamma} \in k\left[\Omega^{\prime}\right]$ by

$$
(\Gamma F)\left(X_{i}\right)=\sum_{\alpha, \beta, \gamma} H_{i, \alpha, \beta, \gamma} X^{\alpha} \otimes X^{\beta} \otimes X^{\gamma} .
$$

Clearly the scheme $D$, resp. $W$, is defined by the equations

$$
\begin{aligned}
T_{i, \alpha, \beta} & =T_{i, \beta, \alpha}, & & \text { all } 1 \leqq i \leqq m \text { and }(\alpha, \beta) \in \Delta^{\prime}, \text { resp. }(\alpha, \beta) \in \Omega^{\prime} ; \\
T_{i, \alpha, \beta} & =0 & & \text { if } X^{\alpha} \otimes X^{\beta} \text { does not satisfy }(P v)_{i} ; \\
H_{i, \alpha, \beta, \gamma} & =0, & & \text { all } 1 \leqq i \leqq m, \text { and }(\alpha, \beta, \gamma) \in \Delta^{\prime \prime}, \text { resp. }(\alpha, \beta, \gamma) \in \Omega^{\prime \prime} .
\end{aligned}
$$


Consider $(F \otimes 1) F\left(X_{i}\right)$; part of this has the form

$$
\sum T_{i, \alpha, \beta}\left\{\prod_{j}\left(X_{j} \otimes 1+1 \otimes X_{j}+\sum T_{j, \gamma, \delta} X^{\nu} \otimes X^{\delta}\right)^{\alpha_{j}}\right\} \otimes X^{\beta} ;
$$

each term of this sum is of the form

$$
T_{i, \alpha, \beta} \cdot \prod_{1 \leqq t \leqq|\alpha|}\left(T_{l, \lambda_{t}, \mu_{t}} X^{\lambda_{t}} \otimes X^{\mu_{t}}\right) \otimes X^{\beta}
$$

(where the question mark indicates some integer, $1 \leqq ? \leqq m$, and where $\left.T_{?, 1,0}=1=T_{2,0,1}\right)$; the monomial in the $T$ 's obtained thus has weight

$$
|\alpha|+|\beta|-1+\sum_{t}\left(\left|\lambda_{t}\right|+\left|\mu_{t}\right|-1\right)=a,
$$

while the corresponding term in the $X$ 's has total degree

$$
\sum_{t}\left|\lambda_{t}\right|+\sum_{t}\left|\mu_{t}\right|+|\beta|=a+1
$$

so each term in the polynomial $H_{i, \alpha, \beta, \gamma}$ has weight $|\alpha|+|\beta|+|\gamma|-1$.

Thus both $D$ and $W$ are defined by homogeneous equations in the weighed variables $T_{i, \alpha, \beta}$ and as $0 \in D(k)$, resp. $0 \in W(k)$ are non-singular points we deduce from the elimination lemma that both $D$ and $W$ are isomorphic to affine space over $k$. This finishes the proof of the first statement of (4.1). Hence Theorem (3.1) is proved, as we have seen (3.2) that $\Sigma \cong \Omega$.

Let $\mathfrak{a} \subset k\left[\Delta^{\prime}\right]$, respectively $\mathfrak{b} \subset k\left[\Omega^{\prime}\right]$ be the ideal defining $D$, resp. $W$. Renaming the variables we obtain: $k\left[\Omega^{\prime}\right]=k\left[T_{1}, \ldots, T_{N}\right]$ and $k\left[\Delta^{\prime}\right]=$ $k\left[T_{1}, \ldots, T_{N}, T_{N+1}, \ldots, T_{N+M}\right]$. We have proved already that there exists a number $n$, with $0 \leqq n \leqq N$, so that

$$
k\left[T_{1}, \ldots, T_{n}\right] \longleftrightarrow k\left[T_{1}, \ldots, T_{N}\right]
$$

The morphism $\rho: D \rightarrow W$ comes from the ringhomomorphism $\varphi$ :

$$
\begin{gathered}
k\left[T_{1}, \ldots, T_{N}\right] \subset k\left[T_{1}, \ldots, T_{N+M}\right] \\
\downarrow \\
k\left[T_{1}, \ldots, T_{n}\right] \cong U=k\left[T_{1}, \ldots, T_{N}\right] / \mathfrak{b} \stackrel{\varphi}{\longrightarrow} k\left[T_{1}, \ldots, T_{N+M}\right] / \mathfrak{a}=B, \\
\operatorname{Spec}(U)=W \stackrel{\rho}{\stackrel{S}{\longrightarrow}} D=\operatorname{Spec}(B), \quad \rho={ }^{\mathfrak{a}} \varphi ;
\end{gathered}
$$

we are done if we can prove that if we apply the elimination lemma to $\mathfrak{a} \subset k\left[\mathrm{~T}_{1}, \ldots, T_{N+M}\right]$, none of the variables $T_{1}, \ldots, T_{n}$ is eliminated: in that case

$$
k\left[T_{1}, \ldots, T_{n}\right] \cong U \rightarrow B \cong k\left[T_{1}, \ldots, T_{n}, T_{N+1}, \ldots, T_{N+m}\right]
$$


for some $m$ with $0 \leqq m \leqq M$ (renumber the variables if necessary); of course in that case every $R$-point of $W$ comes from an $R$-point of $D$. So we have to show: if $T_{i, \alpha, \beta}$ with $(\alpha, \beta) \in \Omega^{\prime}$ appears in the linear term of some $H_{j, \gamma, \delta, \varepsilon}$, with $(\gamma, \delta, \varepsilon) \in \Delta^{\prime \prime}$, then $(\gamma, \delta, \varepsilon) \in \Omega^{\prime \prime}$; but this is clear: computing $(\Gamma F)\left(X_{i}\right)$ we obtain:

$$
\begin{aligned}
\sum T_{i, \alpha, \beta} X^{\alpha} \otimes & X^{\beta} \otimes 1-\sum T_{i, \alpha, \beta} 1 \otimes X^{\alpha} \otimes X^{\beta} \\
+ & \sum T_{i, \alpha, \beta}\left\{\prod_{j}\left(X_{j} \otimes 1+1 \otimes X_{j}+\sum T_{j, \gamma, \delta} X^{\gamma} \otimes X^{\delta}\right)^{\alpha_{j}}\right\} \otimes X^{\beta} \\
& -\sum T_{i, \alpha, \beta} X^{\alpha} \otimes\left\{\prod_{j}\left(X_{j} \otimes 1+1 \otimes X_{j}+\sum T_{j, \gamma, \delta} X^{\gamma} \otimes X^{\delta}\right)^{\beta_{j}}\right\} ;
\end{aligned}
$$

so " $T_{i, \alpha, \beta}$ appears in the linear term of $H_{j, \gamma, \delta, \varepsilon}$ " and $(\alpha, \beta) \in \Omega^{\prime}$ imply that $(\gamma, \delta, \varepsilon) \in \Omega^{\prime \prime}$. Thus we have shown that the variables $T_{n+1}, \ldots, T_{N}$ can be expressed in the variables $T_{1}, \ldots, T_{n}$, that $T_{N+1}, \ldots, T_{N+M}$ depend on $T_{1}, \ldots, T_{n}, T_{N+1}, \ldots, T_{N+m}$, and that the variables $T_{1}, \ldots, T_{n}$ cannot be eliminated. Thus the proof of the proposition is concluded.

Remark. The multiplicative semi-group scheme $\mathbf{A}_{1}^{\times}=\operatorname{Spec}(k[T])$ acts on $k\left[\Delta^{\prime}\right]$ and on $k\left[\Omega^{\prime}\right]$ (use the weights of the variables). Under this action $D$ and $W$ are stable, as their defining equations are homogeneous in weight. In this way we originally proved $W$ to be connected; as $D-\{0\} / \mathbf{G}_{m}$ and $W-\{0\} / \mathbf{G}_{\boldsymbol{m}}$ are projective schemes, it easily follows that $\rho: D(k) \rightarrow W(k)$ is surjective in case $k$ is an algebraically closed field.

Remark. One could ask for the dimension of $W$. It is easy to compute directly the equations for the tangent space at $W(k)$. However we do not see a formula expressing $\operatorname{dim} W$ in terms of $m$ and $\left(v_{1}, \ldots, v_{m}\right)$.

Remark. Let $V$ be the $k$-algebraic scheme such that for every $B \supset k$, $V(B)=\left\{\right.$ all commutative $B$-bialgebra structures on $\left.B\left[\tau_{1}, \ldots, \tau_{m}\right]=E\right\}$; then $V_{\text {red }}=W$, and $V=W$ if and only if $v_{1}=\cdots=v_{m}$.

\section{Conclusions}

Corollary (5.1). Let $k$ be a field of characteristic $p>0$, and let $N$ be a finite commutative $k$-group scheme; $N$ can be lifted to characteristic zero (in the sense of problem (B) of Section 1).

Proof. By (2.2) it suffices to show the result for some $K \supset k$; so we can suppose $k$ to be an algebraically closed field. Then $N=N_{\text {loc }} \times N_{\text {sep }}$ (cf. CGS, 2.14). As a reduced finite group scheme over an algebraically closed field corresponds uniquely to a finite group (cf. CGS, 2.16), it is clear that any separable group scheme can be lifted to characteristic zero (we know $N_{\text {sep }}=\operatorname{Spec}(k \times \cdots \times k)$, take any characteristic zero domain $R$ with a reduction $R \rightarrow k$, choose $M=\operatorname{Spec}(R \times \cdots \times R)$, etc.). As $k$ is supposed to be algebraically closed, hence perfect, $N_{\text {loc }}$ admits a truncation type $v=\left(v_{1}, \ldots, v_{m}\right)$, hence by (3.1) there exists a point 
$w \in W(k)$, where $W$ is an irreducible, smooth $k$-algebraic scheme, and a finite, free group scheme $M \rightarrow W$, such that $N_{\mathrm{loc}} \cong M_{w}$ (i.e. the fibre of $M$ at the point $w$ is isomorphic, as a group scheme, with $N_{\text {loc }}$ ). Next we note there exists a point $u \in W(k)$ such that

$$
\mu_{p \exp \left(v_{1}\right)} \times \cdots \times \mu_{p \exp \left(v_{m}\right)} \cong M_{u} ;
$$

thus the fibre of the morphism $M^{D} \rightarrow W$ over the point $u \in W(k)$ is reduced (by $D$ we denote the dualizing functor associating with each finite flat commutative group scheme its linear, or: Cartier, dual; e.g. compare CGS, p.3). Let $L$ be an algebraic closure of the field of fractions of $U$, where $W=\operatorname{Spec}(U)$. It follows that the group scheme $M_{L}^{D}$ is reduced, so $M_{L}^{D}$ can be lifted to characteristic zero by what is said before, so $M_{L}$ can be lifted to characteristic zero as $D$ commutes with base extension, so by (2.3) it follows that $M \otimes_{U} k \cong M_{w} \cong N_{\text {loc }}$ can be lifted to characteristic zero, and the corollary is proved.

Question. Let $R_{0}$ be a local, artinian ring, and let $N_{0}$ be a finite flat, commutative $R_{0}$-group scheme. Can we lift $N_{0}$ to characteristic zero? In case the rank of $N_{0}$ is prime we can, cf. [13]. However it seems that the methods developed above do not work if $R_{0}$ is not a field.

Corollary (5.2). Let $R$ be a ring in which $p \cdot 1=0$, and let $N=\operatorname{Spec}(E)$ be a commutative $R$-group scheme such that $E$ admits a truncation type $E \cong R\left[\tau_{1}, \ldots, \tau_{m}\right], \tau_{i}^{p \exp \left(v_{i}\right)}=0,1 \leqq i \leqq m$ (e.g. $N$ is any finite, commutative, local group scheme over a perfect field $k=R$ ). There exists a commutative formal Lie group on $m$ parameters with coefficients in $R$, having $N$ as a subgroup scheme (i.e. there exists a commutative formal group

$$
f: R \llbracket X_{1}, \ldots, X_{m} \rrbracket \rightarrow R \llbracket X_{1}, \ldots, X_{m}, Y_{1}, \ldots, Y_{m} \rrbracket
$$

inducing the given comultiplication on $\left.R\left[\tau_{1}, \ldots, \tau_{m}\right]\right)$.

Proof. We take $\mathrm{k}=\mathbf{F}_{p} \subset R$; the $R$-bialgebra $E$ with its truncation type defines a point $e \in W(R)$. We choose a big integer $r$; by (4.1) there exists a point $d \in D(R)$ such that $\rho(d)=e$; by the results of Lazard (cf. the beginning of section 4) any commutative $r$-bud on $m$ parameters $e \in D(R)=\Delta_{m, r}(R) \subset \Lambda_{m, r}(R)$ can be extended to a formal Lie group on the same number of parameters, with coefficients in the same ring. Thus the corollary is proved.

Example (constructed by M.Hazewinkel). There exist non-commutative finite local group schemes on $m$ parameters which cannot be embedded into a formal Lie group on $m$ parameters. Let $\operatorname{char}(k)=p$, $n$ and $m$ are positive integers, and $a, b \in k$. We define

$$
\begin{aligned}
E & =k[\tau] /\left(\tau^{p \exp (n+m)}\right), \\
s(\tau) & =\tau \otimes 1+1 \otimes \tau+a \tau^{p^{n}} \otimes \tau^{p^{m}}+b \tau^{p^{m}} \otimes \tau^{p^{n}} .
\end{aligned}
$$


The $s$ thus defined is associative; it is not cocommutative if we choose $n \neq m$ and $a \neq b$; in that case we have a local bialgebra on one parameter, which cannot be extended to a formal Lie group on one parameter if $k$ is a field, because every one-parameter formal Lie group over $k$ is commutative, cf. [6], and [7], Theorem 1, p. 253.

Remark. By different methods it was proved that any finite commutative group scheme over any field $k$ can be embedded into an irreducible smooth $k$-algebraic group scheme $G$ (cf. CGS, 15.4; cf. [12], in that case we can even take for $k$ a complete local noetherian ring); however in general the dimension of $G$ is much bigger than the number of parameters of $N$ (suppose $N$ to be local); in fact, if the rank of $N$ is $p^{d}$, and $k$ is algebraically closed, an imbedding of $N$ into a $d$-dimensional group variety was constructed. In general a local finite, commutative group scheme on $m$ parameters cannot be embedded into a group variety of dimension $m$ (i.e. $N$ being fixed, none of the formal Lie groups constructed in 5.2 need to be algebraizable), as is shown by the following

Example. Let $k$ be a perfect field of characteristic $p$, and let $N$ be the $k$-group scheme having as Dieudonné-module $W_{\infty}(k)[F, V] /\left(V-F^{2}\right.$, $F^{i}$ ), with $i \geqq 3$; this is a local group scheme on one parameter; it has rank $p^{i}$, the rank of $\operatorname{Ker}\left(p \cdot 1_{N}\right)$ is $p^{3}$ and the rank of $\operatorname{Ker}\left(V_{N}\right)$ is $p^{2}$. If $G$ is an abelian variety of dimension one, the rank of $\operatorname{Ker}\left(p \cdot 1_{G}\right)$ is $p^{2}$, so $N \subset G$ is excluded. As $0 \neq \operatorname{Ker}\left(V_{N}\right)$, the case $N \subset \mathbf{G}_{m}$ is not possible. As $\operatorname{Ker}\left(V_{N}\right) \neq N$, we cannot embed $N$ into a one-dimensional unipotent group-variety $G$ (because any one-dimensional unipotent group variety is killed by $V$ ). Thus the $N$ we have choosen cannot be embedded into a one-dimensional group variety.

Remark. Let $v_{1} \leqq v_{2} \leqq \cdots \leqq v_{m}, \mu_{1} \leqq \mu_{2} \leqq \cdots \leqq \mu_{m}$, with $\mu_{i} \geqq v_{i}$ for $1 \leqq i \leqq m$, and $v_{j}-v_{i} \geqq \mu_{j}-\mu_{i}$ for $1 \leqq i<j \leqq m$; using the methods exposed above, one can show that any $s \in \Omega_{v}(R)$ can be extended to an element $t \in \Omega_{\mu}(R)$; taking $\mu_{1}=a=\mu_{2}=\cdots=\mu_{m}$, and letting $a$ grow, we obtain again (5.2).

\section{References}

1. Cartier, P.: Groupes algébriques et groupes formels. Coll. CBRM, Brussels 1962, $87-111$.

2. Demazure, M., et A. Grothendieck: Schémas en groupes. Sém. géom. algébrique, IHES, 1963-1964. Referred to as SGAD.

3. Grothendieck, A., et J. Dieudonné: Éléments de géométrie algébrique. Publ. Math., IHES. Referred to as EGA.

4. Grothendieck, A.: Séminaire de géométrie algébrique. IHES, 1960. Referred to as SGA

5. Lazard, M.: Lois de groupes et analyseurs. Ann. Sc. Éc. norm. sup. 72, 299-400 (1955).

6. - La non-existence des groupes de Lie formels non abéliens à un paramètre. C. R. Acad. Sci. 239, 942-945 (1954).

23 Inventiones math., Vol. 5 
334 F. Oort et al.: Deformations and Liftings of Finite, Commutative Group Schemes

7. Lazard, M.: Sur les groupes de Lie formels à un paramètre. Bull. Soc. Math. France 83, $251-274$ (1955).

8. Lubin, J., and J. Tate: Formal moduli for one-parameter Lie groups. Bull. Soc. Math. France 94, 49-60 (1966).

9. Mumford, D.: Lectures on curves on an algebraic surface (lecture notes Harvard University, 1964). Princeton Math. Notes 59.

10. Oort, F.: Commutative group schemes. Lecture Notes in Math. 15. Berlin-HeidelbergNew York: Springer 1966. Referred to as CGS.

11. - Algebraic group schemes in characteristic zero are reduced. Inv. Math. 2, 79-80 (1966).

12. - Embedding of finite group schemes into abelian schemes. Mimeographed notes from the advanced science seminar in algebraic geometry, Bowdoin college, summer 1967.

13. Tate, J., and F. Oort: Finite group schemes of prime rank (to appear).

\section{Frans Oort}

Mathematisch Instituut

Nieuwe Achtergracht 121

Amsterdam

The Netherlands
David Mumford

Department Mathematics

Harvard University

2 Divinity Avenue

Cambridge, Mass., USA

(Received February 22, 1968) 